\title{
THE DIAGNOSTIC VALUE OF CONDUCTING VOIDING CYSTOURETHROGRAM IN ALL INFANTS YOUNGER THAN TWO MONTHS AFTER THEIR FIRST URINARY TRACT INFECTION
}

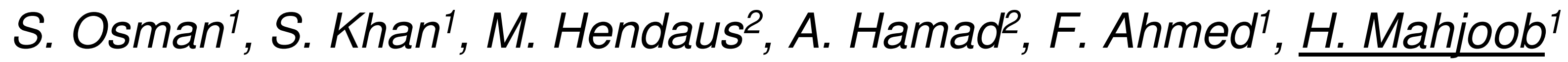 \\ 1-Department of General Pediatrics, Hamad Medical Corporation, Qatar \\ 2-Department of General Pediatrics, Sidra Medicine, Qatar
}

\section{Background and Aims}

This study was conducted to determine the need for Voiding Cystourethrogram (VCUG) in infants below 2 months after their first urinary tract infection (UTI), irrespective of renal ultrasound result (RUS).

\section{Method}

A retrospective study was conducted at a tertiary medical institution in Qatar. Patients below 2 months hospitalized with a first episode of UTI from January 1, 2008 to December 31, 2013 were included.

\section{Results}

RUS was conducted in all the 136 patients (52\% females, $48 \%$ males). Of these 136 patients, 87 (63\%) also had a VCUG done to screen for VUR. The VCUG results were divided into three categories: normal VCUG ( $n=56)$, VCUG with grade 1-2 reflux $(n=11)$ and VCUG with grade $3-5$ reflux $(n=20) .72 .7 \%$ of the 11 patients, who had a grade 1-2 VUR, had a normal RUS, which is often the case with low grade reflux. However, the RUS also failed to pick up any abnormalities in 13 out of the 20 babies (65\%) with grade $3-5$ reflux. Our study showed that RUS had a sensitivity of $32.2 \%$, specificity of $75 \%$, and negative predictive value of $66.7 \%$ with a positive predictive value of $41.7 \%$ to identify VUR, when compared to VCUG in these infants.

\section{Conclusion}

The overall diagnostic accuracy of ultrasound to detect VUR was found to be $59.8 \%$. Our data demonstrates that RUS alone, without a VCUG, might fail to detect even clinically significant VUR i.e. grade 3-5 reflux. Hence imaging after the first UTI should include RUS in combination with VCUG in infants below 2 months.

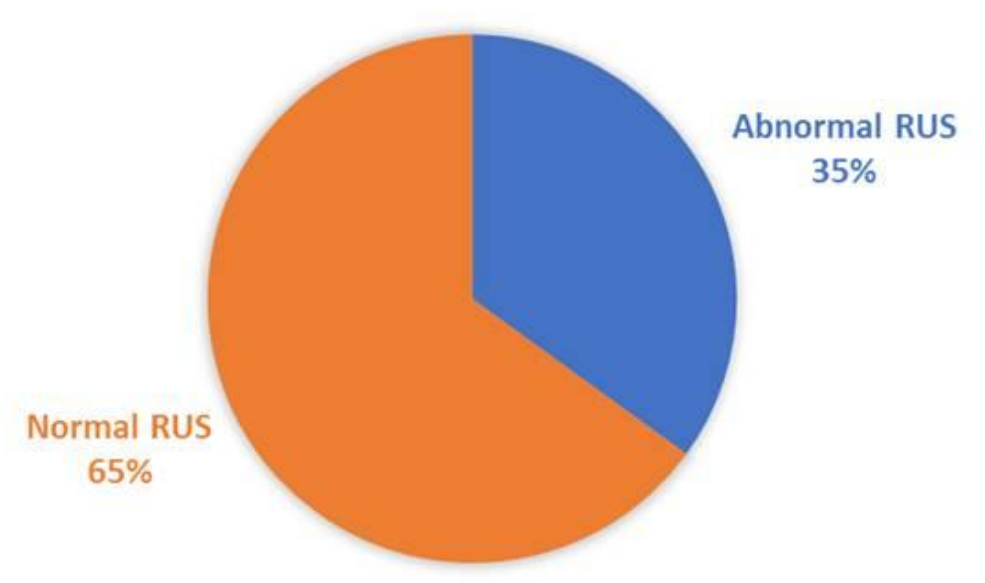

